Pacific Journal of Mathematic 


\section{INDEFINABILITY IN THE ARITHMETIC ISOLIC INTEGERS}

\section{PHILIP OLIN}

This paper is primarily concerned with the theory of the arithmetic isolic integers. The following results are obtained :

(1) No nonfinite member of the arithmetic isolic integers $\Lambda^{*}(A)$ can be defined in $\Lambda^{*}(A)$ even by an infinite number of arithmetic formulas (Theorem 4).

(2) The arithmetic isols $\Lambda(A)$ cannot be defined in $\Lambda^{*}(A)$ even by an infinite number of arithmetic formulas (Theorem 7 ).

(3) We exhibit some nonstandard models of arithmetic contained within $\Lambda^{*}(A)$ (Theorem 10).

The first result above follows from recent work of Nerode in the theory of isols, while the second strengthens his results to obtain the desired conclusion.

The ring of arithmetic isolic integers $\Lambda^{*}(A)$ was introduced by Nerode in [5] where he showed that $\Lambda^{*}(A)$ is elementarily equivalent to a reduced power $Q$ of the ring of integers and where he adapted the method of Feferman and Vaught [1] to $\Lambda^{*}(A)$. In [6] Nerode gave a procedure for finding isols and isolic integers which satisfy extensions to isols of recursively enumerable relations. The work which follows here both uses these results and, in some cases, strengthens them. We use the definitions and notation of [4], [5], and [6].

It is possible that a nice structure theorem for $\Lambda^{*}(A)$ can be proved; Nerode has asked whether $\Lambda^{*}(A)$ is isomorphic to $Q$, the reduced power of the integers just mentioned (see remarks following Corollary 5 below). E. Ellentuck has obtained such a result for the ring of Dedekind finite cardinals in the models of a particular extension of Zermelo Fraenkel set theory without the axiom of choice. However even if such a result is obtained for $\Lambda^{*}(A)$, since $\Lambda(A)$ cannot be defined in $\Lambda^{*}(A)$ by an infinite number of arithmetic formulas, it will still not be possible to arithmetically define in this way the substructure corresponding to $\Lambda(A)$.

1. We adopt the notation of [4], [5], and [6]. As in [5] and [7], $Q=Z^{\omega} / D_{0}$ where $Z$ is the ring of integers and $D_{0}$ the filter of cofinite subsets of $\omega$.

THEOREM 1. Suppose $\left\{\varphi_{i}\right\}_{i<\omega}$ is a collection of arithmetic formulas in the variables $\bar{v}=\left(v_{0}, v_{1}, v_{2}, \cdots\right)$ and the collection is finitely satisfiable in $Q$. Then there exist $A, B \in Q^{\omega}$ such that 
(i) for all $i, Q \vDash \varphi_{i}(A)$

(ii) for all $i, Q \vDash \varphi_{i}(B)$

(iii) for all $j$, if $A_{j} \in E^{*}$ then $A_{j}=B_{j}$ and if $A_{j} \notin E^{*}$ then $B_{j} \notin E^{*}$ and $A_{j} \neq B_{j}$.

We give only a sketch of the proof. In [2] it is shown that $Q$ is $\omega_{1}$-saturated. From this we get $A \in Q^{\omega}$ satisfying (i). $B$ is constructed from $A$. If $A_{j} \in E^{*}, B_{j}=A_{j}$. If $A_{j} \in Q-E^{*}, B_{j}$ is gotten from $A_{j}$ by a permutation of the coordinates. The permutation is simply to ensure that $B_{j} \neq A_{j}$ and, from the Feferman-Vaught method of [1], the permutation of coordinates does not affect satisfaction in $Q$.

Lemma 2. Suppose $\varphi\left(v_{1} \cdots, v_{n}\right)$ is an arithmetic formula with $n$ free variables, $A_{i} \in \Lambda^{*}(A)$ for $1 \leqq i \leqq n$,

$$
A_{1} \notin E^{*}, \quad \text { and } \quad \Lambda^{*}(A) \models \varphi\left(A_{1}, \cdots, A_{n}\right) \text {. }
$$

Then there exist $B_{i} \in \Lambda^{*}(A)$ for $1 \leqq i \leqq n$, with

$$
B_{1} \neq A_{1} \quad \text { and } \quad \Lambda^{*}(A) \vDash \varphi\left(B_{1}, \cdots, B_{n}\right) .
$$

Proof. Assume the conclusion is false. Then we have

$$
\begin{aligned}
& \Lambda^{*}(A) \vDash\left(E x_{1}\right) \cdots\left(E x_{n}\right)\left[\varphi\left(x_{1}, \cdots, x_{n}\right) \wedge\left(y_{1}\right) \cdots\left(y_{n}\right)\right. \\
& \left.\left(\varphi\left(y_{1}, \cdots, y_{n}\right) \rightarrow y_{1}=x_{1}\right)\right] .
\end{aligned}
$$

In [5] Nerode proved that $Q \equiv \Lambda^{*}(A)$. Hence this statement is also true in $Q$. For $i=1$ to $n$, let $A_{i}^{\prime} \in Q$ be the $x_{i}$ whose existence is thus asserted. It follows from Theorem 1 that $A_{1}^{\prime} \in E^{*}$. So the arithmetic sentence $\left(E x_{2}\right) \cdots\left(E x_{n}\right) \varphi\left(A_{1}^{\prime}, x_{2} \cdots, x_{n}\right)$ is true in $Q$ and hence in $\Lambda^{*}(A)$. So by our assumption $A_{1}=A_{1}^{\prime} \in E^{*}$, a contradiction.

As a consequence of the results in $\S 3$ of Nerode [5] we obtain almost at once the following result. Corresponding to any arithmetic formula $\varphi$ there is another formula $\psi$ which is a disjunction of conjunctions of equations and their negations, each such equation being of the form $f_{A^{*}(A)}=0$ with $f$ an arithmetic function whose free variables are among those of $\varphi$, and such that for

$$
X \in \Lambda^{*}(A)^{\omega}, \quad \Lambda^{*}(A) \vDash \varphi(X) \leftrightarrow \Lambda^{*}(A) \vDash \psi(X) .
$$

Lemma 3. Suppose $\left\{\phi_{i}\right\}_{i<\omega}$ is a collection of arithmetic formulas with free variables among $\bar{v}=\left(v_{0}, v_{1}, v_{2}, \cdots\right)$, and suppose any finite subset of this collection is satisfiable in $\Lambda^{*}(A)$. Then the collection is satisfiable in $\Lambda^{*}(A)$. 
Proof. To each $\varphi_{i}$ there corresponds a $\psi_{i}$ as above. Let us write $\psi_{i}$ as $\mathbf{V}_{j \in J_{i}} C_{j}^{i}$ where the $C_{j}^{i}$ 's are the various conjunctions of $\psi_{i}$. Since the $\varphi_{i}$ 's are finitely satisfiable in $\Lambda^{*}(A)$, so are the $\psi_{i}$ 's. We claim there is a $k \in J_{0}$ such that the collection $\left\{\psi_{i}\right\}_{i \in \omega-\{0\}} \cup\left\{C_{k}^{0}\right\}$ is finitely satisfiable in $\Lambda^{*}(A)$. If not then for each $j \in J_{0}$ there is a finite $S_{j} \leqq \omega-\{0\}$ such that $\left\{\psi_{i}\right\}_{i \in S_{j}} \cup\left\{C_{j}^{0}\right\}$ is not satisfiable in $\Lambda^{*}(A)$. Let $S=\cup_{j \in J_{0}} S_{j}$. But $\left\{\psi_{i}\right\}_{i \in S} \cup\left\{\psi_{0}\right\}$ is satisfiable and hence there is some disjunct $C_{k}^{0}$ of $\psi_{0}$ such that $\left\{\psi_{i}\right\}_{i \in S} \cup\left\{C_{k}^{0}\right\}$ is satisfiable and thus $\left\{\psi_{i}\right\}_{i \in S_{k}} \cup\left\{C_{k}^{0}\right\}$ is satisfiable, which is a contradiction. So $\left\{C_{k}^{0}\right\} \cup\left\{\psi_{i}\right\}_{i \in \omega-\{0\}}$ is finitely satisfiable in $\Lambda^{*}(A)$. Apply the same procedure to $\psi_{1}$ in this last collection, getting $\left\{C_{k}^{0}\right\} \cup\left\{C_{k}^{1}\right\} \cup\left\{\psi_{i}\right\}_{i \in \omega-\{0,1\}}$ finitely satisfiable. By induction we obtain in this way a collection $\left\{C^{i}\right\}_{i<\omega}$, with $C^{i}$ one of the disjuncts of $\psi_{i}$, and the collection finitely satisfiable in $\Lambda^{*}(A)$. Simultaneously satisfying this collection would clearly also simultaneously satisfy $\left\{\phi_{i}\right\}_{i<\omega}$. So the problem is now reduced to the following. Given a collection $\left\{f_{\Lambda^{*}(A)}^{i}=0\right\}_{i<\omega} \cup\left\{g_{A^{*}(A)}^{i} \neq 0\right\}_{i<\omega}$, variables among $\bar{v}=\left(v_{0}, v_{1}, v_{2}, \cdots\right)$, each $f$ and $g$ an arithmetic function, and the collection finitely satisfiable in $\Lambda^{*}(A)$ : show that the whole collection is satisfiable in $\Lambda^{*}(A)$.

Let $X_{\omega} E^{*}$ be the subset of $E^{* \omega}$ consisting of the finitely nonzero sequences and let $R^{i}\left(T^{i}\right)$ consist of those $x \in X_{\omega} E^{*}$ such that $f^{i}(x)=0$ $\left(g^{i}(x)=0\right)$. We shall use Theorem 2.1 of Nerode [4], in the arithmetic case. A first application of this theorem enables us to infer from the finite satisfiability of $\left\{f_{\Lambda^{*}(A)}^{i}=0\right\}$ in $\Lambda^{*}(A)$ that the collection $\left\{R^{i}\right\}_{i<\omega}$ generates a proper filter $F^{*}$ in the lattice $L^{*}$ of finitary arithmetic relations (see [6]). So by Theorem 4.7 of [6] in the arithmetic case, there is an $X \in \Lambda^{*}(A)^{\omega}$ such that

$$
F^{*}=\left\{R \in L^{*} \mid X \in R_{A^{*}(A)}\right\} \text {. }
$$

With another application of Theorem 2.1 of [4] we get $X \in R_{A^{*}(A)}^{i}$ if and only if $f_{i^{*}(A)}^{i}(X)=0$. Since for all $i, R^{i} \in F^{*}, X$ satisfies $\left\{f_{\Lambda^{*}(A)}^{i}=0\right\}_{i<\omega}$. Does it also satisfy $\left\{g_{\Lambda^{*}(A)}^{i} \neq 0\right\}$ ? Suppose not; then there is some $i$ such that $g_{A^{*}(A)}^{i}(X)=0$. Then, as above, $X \in T_{A^{*}(A)}^{i}$. Hence $T^{i} \in F^{*}$. So there exist $R^{i_{0}}, \cdots, R^{i_{n}}$ such that

$$
R^{i_{0}} \cap \cdots \cap R^{i_{n}} \subseteq T^{i}
$$

Thus $X_{\omega} E^{*}=\overline{R^{i_{0}}} \cup \cdots \cup \overline{R^{i_{n}}} \cup T^{i}$. Yet another application of Theorem 2.1 of [4] shows that the set $\left\{f_{A^{*}(A)}^{i_{0}}=0, \cdots, f_{A^{*}(A)}^{i_{n}}=0, g_{A^{*}(A)}^{i} \neq 0\right\}$ cannot be satisfied in $\Lambda^{*}(A)$, which contradicts the hypothesis. Thus $X$ satisfies $\left\{f_{A^{*}(A)}^{i}=0\right\}_{i<\omega} \cup\left\{g_{A^{*}(A)}^{i} \neq 0\right\}_{i<\omega}$, as required.

The next theorem is the analogue in $\Lambda^{*}(A)$ of Theorem 1 . 
THEOREM 4. Suppose $\left\{\varphi_{i}\right\}_{i<\omega}$ is a collection of arithmetic formulas in the variables $\bar{v}=\left(v_{0}, v_{1}, v_{2}, \cdots\right)$ and the collection is finitely satisfiable in $\Lambda^{*}(A)$. Then three exist $X, Y \in \Lambda^{*}(A)^{\omega}$ such that

(i) for all $i, \Lambda^{*}(A) \vDash \varphi_{i}(X)$

(ii) for all $i, \Lambda^{*}(A) \vDash \varphi_{i}(Y)$

(iii) for all $j$, if $X_{j} \in E^{*}$ then $X_{j}=Y_{j}$ and if $X_{j} \notin E^{*}$ then $Y_{j} \notin E^{*}$ and $X_{j} \neq Y_{j}$.

Proof. As in Lemma 3 we obtain $X^{\prime} \in \Lambda^{*}(A)^{\omega}$ and a collection $K_{v}=\left\{f_{\Lambda^{*}(A)}^{i}(v)=0\right\}_{i<\omega} \cup\left\{g_{\Lambda^{*}(A)}^{i}(v) \neq 0\right\}_{i<\omega}$ which $X^{\prime}$ satisfies and if any $X^{\prime \prime}$ satisfies $K_{v}$ than $X^{\prime \prime}$ satisfies (i) above. For the variables $\bar{u}=\left(u_{0}, u_{1}, u_{2}, \cdots\right)$, let $K_{u}$ be the collection gotten from $K_{v}$ simply by replacing each $v_{i}$ by $u_{i}$. Then $\left(X^{\prime}, X^{\prime}\right) \in\left(\Lambda^{*}(A)^{\omega}\right)^{2}$ satisfies $K_{v} \cup K_{u}$ (where $\varphi\left(Z, Z^{\prime}\right.$ ) for $\left(Z, Z^{\prime}\right) \in\left(\Lambda^{*}(A)^{\omega}\right)^{2}$ means replace $v_{i}$ by $Z_{i}$ and $u_{i}$ by $Z_{i}^{\prime}$ ). Using the lattice $L^{*}$ of finitary arithmetic relations on $\left(X_{\omega} E^{*}\right)^{2}$, let $R_{v}^{i}$ and $R_{u}^{i}$ denote respectively the arithmetic relations corresponding to $f^{i}(v)=0$ and $f^{i}(u)=0$. Proceeding in the usual way (see [6]) we let $F^{*}$ be the filter generated by the $R_{v}^{i}$ 's and $R_{u}^{i}$ 's and get $(X, Y) \in\left(\Lambda^{*}(A)^{\omega}\right)^{2}$. With methods like those of Lemma 3 we show that $X$ satisfies (i) above and $Y$ satisfies (ii). Because for each $i, X_{i}^{\prime}=a$ for $a$ in $E^{*}$ if and only if

$$
\left\{(v, u) \in\left(X_{\omega} E^{*}\right)^{2} \mid v_{i}=a\right\} \text { and }\left\{(v, u) \in\left(X_{\omega} E^{*}\right)^{2} \mid u_{i}=a\right\}
$$

are in $F^{*}$, it follows that if $X_{i}^{\prime}$ is in $E^{*}$ then $X_{i}^{\prime}=X_{i}=Y_{i}$ and if $X_{i}^{\prime} \notin E^{*}$ then $X_{i} \notin E^{*}$ and $Y_{i} \notin E^{*}$. Assume $i$ is such that $X_{i}^{\prime} \notin E^{*}$. We claim $X_{i} \neq Y_{i}$. Suppose not. Let $T=\left\{(v, u) \in\left(X_{\omega} E^{*}\right)^{2} \mid v_{i}=u_{i}\right\}$. Then $(X, Y) \in T_{A^{*}(A)}$ and hence $T \in F^{*}$. Proceeding as in Lemma 3 there exist $f^{i_{1}}, \cdots, f^{i_{n}}, f^{j_{1}}, \cdots, f^{j_{m}}$ such that

$$
\left\{f_{\Lambda^{*}(A)}^{i_{1}}(v)=0, \cdots f_{\Lambda^{*}(A)}^{i_{n}}(v)=0, f_{\Lambda^{*}(A)}^{j_{1}}(u)=0, \cdots, f_{\Lambda^{*}(A)}^{j_{m}}(u)=0, v_{i} \neq u_{i}\right\}
$$

cannot be satisfied in $\Lambda^{*}(A)$. Thus for an appropriate finite set of $\varphi$ 's we have that $\Lambda_{j} \varphi_{j}(v) \wedge \Lambda_{k} \varphi_{k}(u) \wedge\left(v_{i} \neq u_{i}\right)$ cannot be satisfied in $\Lambda^{*}(A)$. But $\left(X^{\prime}, X^{\prime}\right)$ satisfies $\Lambda_{j} \varphi_{j}(v) \wedge \Lambda_{k} \varphi_{k}(u)$ and $X_{i}^{\prime} \notin E^{*}$. So by Lemma 2 we can find $X^{\prime \prime} \in \Lambda^{*}(A)^{\omega}$ such that $\left(X^{\prime \prime}, X^{\prime \prime}\right)$ satisfies $\bigwedge_{j} \varphi_{j}(v) \wedge \bigwedge_{k} \varphi_{k}(u)$ and $X_{i}^{\prime \prime} \neq X_{i}^{\prime}$. But then $\left(X^{\prime}, X^{\prime \prime}\right)$ satisfies

$$
\bigwedge_{j} \varphi_{j}(v) \wedge \Lambda_{k} \varphi_{k}(u) \wedge\left(v_{i} \neq u_{i}\right),
$$

a contradiction. Thus $X_{i} \neq Y_{i}\left(T \notin F^{*}\right)$, completing the theorem.

Corollary 5. No member of $\Lambda^{*}(A)-E^{*}$ can be defined in $\Lambda^{*}(A)$ even by an infinite number of arithmetic formulas.

We conclude this section with another application of the methods 
used above. Nerode has asked whether $Q \cong \Lambda^{*}(A)$. (In [5] he showed $Q \equiv \Lambda^{*}(A)$ and both have power $2^{\aleph_{0}}$.) The answer is not trivially yes because by the following example, pointed out to the author by M. Morley, the theory of $Q$ (and hence of $\Lambda^{*}(A)$ ) is not categorical in any infinite power. Let $p_{0}, p_{1}, p_{2}, \ldots$ be a sequence of distinct positive primes, and $q_{0}, q_{1}, q_{2}, \ldots$ a list of all the other positive primes. Consider $\left\{p_{i} \text { divides } x\right\}_{i<\omega} \cup\left\{q_{i} \text { does not divide } x\right\}_{i<\omega}$. This countable collection of arithmetic formulas is clearly finitely satisfiable in $Q$. Since $Q$ is $\omega_{1}$-saturated, the collection is simultaneously satisfiable in $Q$. But there are $2^{\aleph_{0}}$ such types realized in $Q$. So by a result of Ehrenfeucht, the theory of $Q$ is not categorical in any infinite power.

In [7] it is shown that, assuming the continuum hypothesis, $Q \cong \Lambda^{*}(A)$ if and only if $\Lambda^{*}(A)$ is saturated. Using the method of Lemma 3 it can be shown that $\Lambda^{*}(A)$ is saturated if and only if for every collection $\left\{f_{A^{*}(A)}^{i}=0\right\}_{i<\omega} \cup\left\{g_{A^{*}(A)}^{i} \neq 0\right\}_{i<\omega}$, where the $f^{\prime}$ s and $g^{\prime}$ s are arithmetic functions having exactly one free variable and possibly constants from $\Lambda^{*}(A)$, if the collection is finitely satisfiable in $\Lambda^{*}(A)$ then it is satisfiable in $\Lambda^{*}(A)$.

2. We shall prove shortly that $\Lambda(A)$ cannot be defined in $\Lambda^{*}(A)$ by an infinite number of formulas. In particular, one formula will not define $\Lambda(A)$ within $\Lambda^{*}(A)$. The former result requires a strengthening of the proof of Theorem 3.1 of [6]. But the latter can be proved as a corollary of that theorem.

THEOREM 6. $\Lambda(A)$ cannot be defined in $\Lambda^{*}(A)$ by means of one arithmetic formula (and hence by means of a finite number of such formulas).

Proof. We prove something a little stronger. Suppose $\varphi(x)$ is an arithmetic formula of one free variable, $X \in \Lambda(A)-E$, and $\Lambda^{*}(A) \vDash \varphi(X)$. We shall find $Y \in \Lambda^{*}(A)-(\Lambda(A) \cup-\Lambda(A))$ such that $\Lambda^{*}(A) \vDash \varphi(Y)$.

By the remark preceding Lemma 3, we have

$$
f_{A^{*}(A)}^{1}(x)=0 \wedge \cdots \wedge f_{A^{*}(A)}^{n}(x)=0 \wedge g_{A^{*}(A)}^{1}(x) \neq 0 \wedge \cdots \wedge g_{A^{*}(A)}^{m}(x) \neq 0
$$

satisfied by $X$, and if any $X^{\prime} \in \Lambda^{*}(A)$ satisfies this conjunction then $\Lambda^{*}(A) \vDash \varphi\left(X^{\prime}\right)$. In particular for each $j, 1 \leqq j \leqq m$,

$$
f_{\Lambda^{*}(A)}^{1}(X)=0 \wedge \cdots \wedge f_{\Lambda^{*}(A)}^{n}(X)=0 \wedge g_{\Lambda^{*}(A)}^{j}(X) \neq 0 .
$$

Since $X \in A(A)-E$, we can apply the first part of Theorem 11.1 of [3] to get the existence of an infinite number of members of $E$ which are solutions in $E^{*}$ to 


$$
\left.{ }^{*}\right) f^{1}(x)=0 \wedge \cdots \wedge f^{n}(x)=0 \wedge g^{j}(x) \neq 0 .
$$

We can now find a finite set $S, S \subseteq E^{*}$, cardinality of $S \geqq 2$, such that for each $j, 1 \leqq j \leqq m$, there is an $a_{j} \in S$ satisfying (*) in $E^{*}$. Of course $S \in L^{*}$ (see [6], using only one coordinate). Let $F^{*}$ be the filter of $L^{*}$ generated by $S$. By Theorem 4.7 of [6] there is a $Y \in \Lambda^{*}(A)$ such that $F^{*}=\left\{R \in L^{*} \mid Y \in R_{\Lambda^{*}(A)}\right\}$. Since $S \in F^{*}, Y \in S_{\Lambda^{*}(A)}$. For each $i, 1 \leqq i \leqq n$, we also have $S \subseteq\left\{x \in E^{*} \mid f^{i}(x)=0\right\}=T^{i}$. So $Y \in T_{\Lambda^{*}(A)}^{i}$. Again by Theorem 2.1 of [4] we have $f_{\Lambda^{*}(A)}^{i}(Y)=0$, $1 \leqq i \leqq n$. We claim $g_{\Lambda^{*}(A)}^{j}(Y) \neq 0,1 \leqq j \leqq m$. Say, for some $j$, $g_{\Lambda^{*}(A)}^{j}(Y)=0$. So for $U^{j}=\left\{x \in E^{*} \mid g^{j}(x)=0\right\}$, again by Theorem 2.1 of [4], $Y \in U_{A^{*}(A)}^{j}$. Hence $U^{j} \in F^{*}$ and $U^{j} \supseteqq S$. Thus

$$
E^{*} \vDash(x)\left(x \notin S \vee g^{j}(x)=0\right) .
$$

Again by Theorem 2.1, $\Lambda^{*}(A) \vDash(X)\left(X \notin S_{A^{*}(A)} \vee g_{A^{*}(A)}^{j}(X)=0\right)$. But $a_{j} \in \Lambda^{*}(A), a_{j} \in S_{A^{*}(A)}$ and $g_{A^{*}(A)}^{j}\left(a_{j}\right) \neq 0$, a contradiction. Thus for all $j, 1 \leqq j \leqq m, g_{A^{*}(A)}^{j}(Y) \neq 0$. Hence $\Lambda^{*}(A) \vDash \varphi(Y)$. We claim $Y \notin E^{*}$. If $Y=a \in E^{*}$ then, for $U=\{a\}, Y \in U_{A^{*}(A)}$ and hence $U \in F^{*}$ and $U \supseteqq S$ which contradicts $S$ having cardinality $\geqq 2$. So $Y \notin E^{*}$. But $Y \in S_{A^{*}(A)}$. Now by Corollary 5.10 of [4] (in the arithmetic case), $Y \in \Lambda^{*}(A)-(\Lambda(A) \cup-\Lambda(A))$.

The next theorem is the major result of this paper. We use the definitions and notation of [6] but always in the arithmetic rather than the recursive case. Further, for simplicity we take $L^{*}$ to be the lattice of arithmetic subsets of $E^{*}$ (since only one variable is needed) and $L$ the lattice of arithmetic subsets of $E^{2}$. Consequently we ignore the notion of "support".

THEOREM 7. The arithmetic isols $\Lambda(A)$ cannot be defined in the arithmetic isolic integers $\Lambda^{*}(A)$ by an infinite number of arithmetic formulas.

Proof. We prove a slightly stronger result. Suppose $\left\{\phi_{i}\right\}_{i<\omega}$ are arithmetic formulas of one free variable, $X \in \Lambda(A)-E$, and for each $i, \Lambda^{*}(A) \vDash \varphi_{i}(X)$. Then we will find $Y \in \Lambda^{*}(A)-(\Lambda(A) \cup-\Lambda(A))$ such that for each $i, \Lambda^{*}(A) \vDash \varphi_{i}(Y)$.

By the remark preceding Lemma 3 we get a collection

$$
\left\{f_{\Lambda^{*}(A)}^{j}(x)=0\right\}_{i<\omega} \cup\left\{g_{A^{*}(A)}^{j}(x) \neq 0\right\}_{i<\omega},
$$

with the $f^{j}$ 's and $g^{j}$ 's arithmetic functions of one variable, such that $X$ satisfies the collection and if any $X^{\prime} \in \Lambda^{*}(A)$ satisfies the collection then for each $i, \Lambda^{*}(A) \vDash \varphi_{i}\left(X^{\prime}\right)$. Let $T^{j} \subseteq E$ be the set of nonnegative integer solutions to $f^{j}(x)=0$. Since $f_{d^{*}(A)}^{j}(X)=0$ for all $j$, the intersection of any finite number of $T^{j}$ 's is infinite (see [3]). Let 
$F^{*}$ be the filter in $L^{*}$ consisting of all $R \in L^{*}$ such that $R$ contains the intersection of some finite number of $T^{j}$ 's, except perhaps for a finite set. If $U^{j}$ is the set of all integer solutions to $g^{j}(x)=0$ then $U^{j} \notin F^{*}$ because, by results of [3] again, since $X$ satisfies every $f_{i^{*}(A)}^{j}(x)=0$ and also this particular $g_{A^{*}(A)}^{j}(x) \neq 0$, we have that every finite intersection of $T^{j}$ 's contains an infinite subset of $E$ not in $U^{j}$. Let $R^{0}, R^{1}, R^{2}, \cdots$ be an enumeration of $F^{*}$ and $W^{0}, W^{1}, W^{2}, \cdots$ an enumeration of $L^{*}-F^{*}$. As in Theorem 4.7 of [6], let $F=\left\{R^{\wedge} \mid R \in F^{*}\right\}$; $F$ is a realizability filter in $L$ enumerated by $\left(R^{0}\right)^{\wedge},\left(R^{1}\right)^{\wedge},\left(R^{2}\right)^{\wedge}, \cdots$. We can now prove a lemma essentially the same as Lemma 3.3 of [6], but using $\left(R^{i}\right)^{\wedge}$ in place of $T^{i},\left(W^{i}\right)^{\wedge}$ in place of $S^{i}$, and $\left(L^{*}-F^{*}\right)^{\wedge}$ in place of $L-F$. (Note that $\left\{\left(W^{i}\right)^{\wedge}\right\}_{i<\omega}$ is not an enumeration of $L-F$.) Assume this has been done. (The same proof will work.)

We now wish to prove a lemma corresponding to Lemma 3.2 of [6] but with a stronger conclusion. So we shall define inductively (two at a time) $\left\{x^{i}\right\}_{i<\omega}, x^{i} \in E^{2}$. Let $P_{0}, P_{1}, P_{2}, \cdots$ be an enumeration of the one-one partial arithmetic functions of one variable. We shall also inductively define a set $G \subseteq E$ which will contain "integers to be avoided."

From the definition of $F^{*}$ it follows that for each $m<\omega$, $\left(R^{0} \cap \cdots \cap R^{m}\right)-W^{m}$ contains an infinite subset of $E$. We assume that $x^{0}, x^{1}, \cdots, x^{2 n-1}$ have been defined, that $G^{n-1}$ is the finite part of $G$ defined so far, and that for any index $s$ of the form $\left(x^{0}, b^{0}\right),\left(x^{1}, b^{1}\right), \cdots,\left(x^{2 n-1}, b^{2 n-1}\right)$ we have $\left(\alpha_{s}\right)_{0} \cap G^{n-1}=\varnothing . \quad\left(G^{-1}=\varnothing\right)$. Choose $z \in\left(R^{0} \cap \cdots \cap R^{2 n}\right)-W^{2 n}$ and $z^{\prime} \in\left(R^{0} \cap \cdots \cap R^{2 n+1}\right)-W^{2 n+1}$ such that $z>z^{\prime}$. These choices are possible because these sets contain an infinite number of positive integers. Define $x^{2 n} \in E^{2}$ to satisfy: (i) $x_{0}^{2 n}-x_{1}^{2 n}=z$, (ii) $\max \left(x_{0}^{2 n-1}, x_{1}^{2 n-1}\right)<\min \left(x_{0}^{2 n}, x_{1}^{2 n}\right)$, and (iii) for any index $s$ with an initial segment of the form $\left(x^{0}, b^{0}\right), \cdots$, $\left(x^{2 n}, b^{2 n}\right)$, we have $\left(\alpha_{s}\right)_{0} \cap G^{n-1}=\varnothing$. Taking into account the induction hypothesis on $G^{n-1}$, such a $x^{2 n}$ exists since there are an infinite number of members of $E^{2}$ satisfying (i) and (ii) and since $G^{n-1}$ is finite, almost all of these satisfy (iii). Now let $V=\mathrm{U}_{s}\left(\alpha_{s}\right)_{0}$ where the union is taken over all indexes $s$ of the form $\left(x^{0}, b^{0}\right), \cdots,\left(x^{2 n}, b^{2 n}\right)$. Then there exists $x^{2 n+1} \in E^{2}$ such that: (i) $x_{0}^{2 n+1}-x_{1}^{2 n+1}=z^{\prime}$, (ii) $\max \left(x_{0}^{2 n}, x_{1}^{2 n}\right)<\min \left(x_{0}^{2 n+1}, x_{1}^{2 n+1}\right)$, and (iii) for any index $s$ of the form $\left(x^{0}, b^{0}\right), \cdots,\left(x^{2 n+1}, b^{2 n+1}\right)$ we have $P_{n}\left(\left(\beta_{s}\right)_{1}\right) \cap V=\varnothing$. Again such a $x^{2 n+1}$ exists because $V$ is a finite set and of the infinite number of ways to satisfy (i) and (ii), almost all will satisfy (iii). And we have $x^{2 n} \in\left(R^{0}\right)^{\wedge} \cap \cdots \cap\left(R^{2 n}\right)^{\wedge}-\left(W^{2 n}\right)^{\wedge}, x^{2 n+1} \in\left(R^{0}\right)^{\wedge} \cap \cdots \cap\left(R^{2 n+1}\right)^{\wedge}-\left(W^{2 n+}\right)^{\wedge}$. Since $z>z^{\prime}$, we have $x_{0}^{2 n}-x_{1}^{2 n}>x_{0}^{2 n+1}-x_{1}^{2 n+1}$ and thus

$$
x_{1}^{2 n+1}-x_{1}^{2 n}>x_{0}^{2 n+1}-x_{0}^{2 n} \text {. }
$$

Let $s$ be an index of the form $\left(x^{0}, b^{0}\right), \cdots,\left(x^{2 n+1}, b^{2 n+1}\right)$. Then $\left(\beta_{s}\right)_{1}$ 
has cardinality $x_{1}^{2 n+1}-x_{1}^{2 n}$ and $\left(\beta_{s}\right)_{0}$ has the smaller cardinality $x_{0}^{2 n+1}-x_{0}^{2 n}$. For this $s$ three cases may then occur.

Case I. $P_{n}$ is not defined on all of $\left(\beta_{s}\right)_{1}$.

Case II. $P_{n}$ is defined on all of $\left(\beta_{s}\right)_{1}$ and $P_{n}\left(\left(\beta_{s}\right)_{1}\right) \subseteq \mathrm{U}_{r}\left(\beta_{r}\right)_{0}$ where the union is taken over all indexes $r$ of the form $\left(x^{0}, b^{0}\right), \cdots$, $\left(x^{2 n+1}, b^{2 n+1}\right)$. In this case, since $P_{n}\left(\left(\beta_{s}\right)_{1}\right)$ has larger cardinality than $\left(\beta_{s}\right)_{0}$, there is an $r \neq s$ and an integer $u$ such that $u \in P_{n}\left(\left(\beta_{s}\right)_{1}\right) \cap\left(\beta_{r}\right)_{0}$.

Case III. Cases I and II do not hold. In this case, again since $P_{n}\left(\left(\beta_{s}\right)_{1}\right)$ has larger cardinality than $\left(\beta_{s}\right)_{0}$, there is a

$$
u \in P_{n}\left(\left(\beta_{s}\right)_{1}-\mathrm{U}_{r}\left(\beta_{r}\right)_{0} .\right.
$$

Define $G^{n}$ to consist of $G^{n-1}$ together with the $u$ 's which come from indexes $s$ satisfying Case III. Then for any index $s$ of the form $\left(x^{0}, b^{0}\right), \cdots,\left(x^{2 n+1}, b^{2 n+1}\right)$ we have $\left(\alpha_{s}\right)_{0} \cap G^{n}=\varnothing$ (using part (iii) of the definition of $\left.x^{2 n+1}\right)$. This completes the inductive definitions of $\left\{x_{i}\right\}_{i<\omega}$ and $G$. We have at least the conclusions of Lemma 3.2 of [6] with $\left(R_{i}\right)^{\wedge}$ replacing $T^{i},\left(W^{i}\right)^{\wedge}$ replacing $S^{i}$, and $\left(L^{*}-F^{*}\right)^{\wedge}$ replacing $L-F$.

Proceeding now as in [6] we obtain the infinite sequence $\left(x^{0}, y^{0}\right)$, $\left(x^{1}, y^{1}\right), \cdots$ whose initial segments are the indexes

$$
t_{d}=\left(x^{0}, y^{0}\right), \cdots,\left(x^{d}, y^{d}\right),
$$

and such that $\alpha=\alpha_{t_{0}} \vee \alpha_{t_{1}} \vee \cdots$. Let $Y=\left\langle\alpha_{0}\right\rangle-\left\langle\alpha_{1}\right\rangle$. We can now prove (as in [6]) lemmas corresponding to Lemmas 3.4 and 3.6 of [6]. Assume this has been done. We claim $\alpha_{0}$ and $\alpha_{1}$ are arithmetically isolated sets. If one of them is not, then proceeding as in Lemma 3.5 of [6] we could show that $\alpha_{0}$ and $\alpha_{1}$ are both arithmetically enumerable. Let $c_{0}, c_{1}, c_{2}, \cdots$ and $e_{0}, e_{1}, e_{2}, \cdots$ be arithmetic enumerations of $\alpha_{0}$ and $\alpha_{1}$ respectively. Let $H$ consist of all pairs of sets of the form

$$
\left(\left\{c_{0}, \cdots, c_{2 n+2}\right\},\left\{e_{0}, \cdots, e_{2 n}\right\}\right), \quad n=0,1,2, \cdots .
$$

Then $\alpha$ is attainable from the arithmetic $\{2\}$-frame $H$. But $\{2\} \notin F^{*}$. So $\{2\}=W^{i}$ for some $i$ and by our version of Lemma 3.4 of [6], $\alpha$ could not be attainable from $H$, a contradiction. So

$$
Y=\left\langle\alpha_{0}\right\rangle-\left\langle\alpha_{1}\right\rangle \in \Lambda^{*}(A) \text {. }
$$

We wish to show $Y \notin \Lambda(A) \cup-\Lambda(A)$. This is equivalent to showing that $\left\langle\alpha_{0}\right\rangle \not\left\langle\alpha_{1}\right\rangle$ and $\left\langle\alpha_{1}\right\rangle \not\left\langle\alpha_{0}\right\rangle$. Suppose $\left\langle\alpha_{0}\right\rangle \leqq\left\langle\alpha_{1}\right\rangle$. If 
$W$ is the set of nonpositive integers then $W \notin F^{*}$ and so $W=W^{i}$ for some $i$. But $W^{\wedge}=\left\{\left(x_{0}, x_{1}\right) \in E^{2} \mid x_{0} \leqq x_{1}\right\}$, and since $\left\langle\alpha_{0}\right\rangle \leqq\left\langle\alpha_{1}\right\rangle$ we must have $\left\langle\alpha_{0}\right\rangle-\left\langle\alpha_{1}\right\rangle \in W_{A^{*}(A)}$, a contradiction. Now assume $\left\langle\alpha_{0}\right\rangle \geqq\left\langle\alpha_{1}\right\rangle$. So there is a one-one partial arithmetic function of one variable, call it $P_{m}$, such that $P_{m}$ is defined on $\alpha_{1}$ and $P_{m}\left(\alpha_{1}\right) \subseteq \alpha_{0}$. Recall the definitions of $x^{2 m}$ and $x^{2 m+1}$. For the index $t_{2 m+1}$ Case I could not have held since $P_{m}$ is defined on all of $\alpha_{1}$. If Case II held then we have the $u$ as described there. But by property (3.10) of [6] it follows that although $u \in P_{m}\left(\alpha_{1}\right), u$ could not be in $\alpha_{0}$. So Case II could not have held and Case III must have held. So there is a $u \in P_{m}\left(\alpha_{1}\right) \cap G$. But by the construction of $G$, any $u \in G$ could not be in any $\left(\alpha_{t_{d}}\right)_{0}$ and thus $u \notin \alpha_{0}$, a contradiction. So

$$
\left\langle\alpha_{0}\right\rangle \nsupseteq\left\langle\alpha_{1}\right\rangle \text {, and } Y \in \Lambda^{*}(A)-(\Lambda(A) \cup-\Lambda(A)) \text {. }
$$

Now by Theorem 2.1 of [4] (arithmetic case),

$$
Y \in T_{\Lambda^{*}(A)}^{j} \rightarrow f_{\Lambda^{*}(A)}^{j}(Y)=0 .
$$

But $T^{j} \in F^{*}$, and thus $Y \in T_{A^{*}(A)}^{j}$. So for all $j<\omega, f_{A^{*}(A)}^{j}(Y)=0$. Suppose $g_{\Lambda^{*}(A)}^{j}(Y)=0$. By the same theorem, $Y \in U_{A^{*}(A)}^{j} \leftrightarrow g_{A^{*}(A)}^{j}(Y)=0$. So $Y \in U_{A^{*}(A)}^{j}$. But we showed $U^{j} \notin F^{*}$; thus $U^{j}$ is some $W^{i}$ and so $Y \notin U_{A^{*}(A)}^{j}$. Hence $g_{A^{*}(A)}^{j}(Y) \neq 0$ and the proof is complete.

COROLlaRY 8. If $F^{*}$, a filter in $L^{*}$, cannot be generated by a singleton set, then there is a $Y \in \Lambda^{*}(A)-(\Lambda(A) \cup-\Lambda(A)$ ) which realizes $F^{*}$.

Proof. First of all, if $F^{*}$ is generated by some singleton set $\{a\}$ for $a \in E^{*}$, then by Theorem 4.7 of [6], $a$ is the one and only member of $\Lambda^{*}(A)$ which realizes $F^{*}$. Now assume $F^{*}$ cannot be generated by a singleton set. Again by Theorem 4.7 of [6], there is an $X \in \Lambda^{*}(A)$ which realizes $F^{*}$. If $X \in E^{*}$ it would follow that $F^{*}$ is generated by $\{X\}$. So $X \in \Lambda^{*}(A)-E^{*}$. If

$$
X \in \Lambda^{*}(A)-(\Lambda(A) \cup-\Lambda(A)),
$$

we would be done. If not, then using this $X$ we can proceed as in the theorem to get $Y \in \Lambda^{*}(A)-(\Lambda(A) \cup-\Lambda(A))$ realizing $F^{*}$.

We remark that the corollary holds also in the recursive case.

3. In this section we prove the analogue for $\Lambda^{*}(A)$ of one of the major results of [7].

Definition 9. A subset $S$ of $\Lambda^{*}(A)$ is said to be indecomposable if for every arithmetic relation $R\left(x_{1}, \cdots, x_{n}\right)$ and every $X_{1}, \cdots, X_{n} \in S$, 
either $\left(X_{1}, \cdots, X_{n}\right) \in R_{A^{*}(A)}$ or $\left(X_{1}, \cdots, X_{n}\right) \in \bar{R}_{\Lambda^{*}(A)}(\bar{R}$ is the complement of $R$ ).

If we apply Theorem 4.7 of [6], using just one variable and taking an ultrafilter $F^{*}$, then the $X \in \Lambda^{*}(A)$ so realized is indecomposable (that is, $\{X\}$ is indecomposable). So indecomposable subsets of $\Lambda^{*}(A)$ exist, and maximal indecomposable subsets properly contain $E^{*}$.

THEOREM 10. Maximal indecomposable subsets of $\Lambda^{*}(A)$ are proper elementary extensions of $E^{*}$.

Proof. Let $S$ be a maximal indecomposable subset. By the remarks above we need only show that $S$ is an elementary extension of $E^{*}$.

We shall first show that $S$ is closed under arithmetic functions extended to $\Lambda^{*}(A)$. Suppose $X_{1}, \cdots, X_{n} \in S$ and $f\left(x_{1}, \cdots, x_{n}\right)$ is an arithmetic function. Let $f_{A^{*}(A)}\left(X_{1}, \cdots, X_{n}\right)=X \in \Lambda^{*}(A)$. Consider $S^{\prime}=S \cup\{X\}$. If it is not indecomposable, there are $Y_{1}, \cdots, Y_{m} \in S$ and an arithmetic relation $R\left(x_{1}, \cdots, x_{m+1}\right)$ such that

$$
\left(X, Y_{1}, \cdots, Y_{m}\right) \notin R_{d^{*}(A)} \cup \bar{R}_{A^{*}(A)} \text {. }
$$

The following statement is true in $E^{*}:\left(x_{1}\right) \cdots\left(x_{n}\right)(x)\left(y_{1}\right) \cdots\left(y_{m}\right)$

$$
\left[f\left(x_{1}, \cdots, x_{n}\right)=x \wedge R\left(f\left(x_{1}, \cdots, x_{n}\right), y_{1}, \cdots, y_{m}\right) \rightarrow R\left(x, y_{1}, \cdots, y_{m}\right)\right] .
$$

And, of course, the same statement is true with $R$ replaced by $\bar{R}$. Since $f$ is an arithmetic function and $R$ and $\bar{R}$ are arithmetic relations we can apply Theorem 2.1 (ii) of [4] in the arithmetic case. This gives :

$$
\Lambda^{*}(A) \vDash \sim R_{i^{*}(A)}\left(X, Y_{1}, \cdots, Y_{m}\right) \rightarrow \sim R_{\Lambda^{*}(A)}\left(f_{d^{*}(A)}\left(X_{1}, \cdots, X_{n}\right), Y_{1}, \cdots, Y_{m}\right)
$$

and the same statement with $R$ replaced by $\bar{R}$. Define an arithmetic relation $R^{\prime}\left(x_{1}, \cdots, x_{n}, y_{1}, \cdots, y_{m}\right) \leftrightarrow R\left(f\left(x_{1}, \cdots, x_{n}\right), y_{1}, \cdots, y_{m}\right)$. (Note that $\bar{R}^{\prime}\left(x_{1}, \cdots, x_{n}, y_{1}, \cdots, y_{m}\right) \leftrightarrow \bar{R}\left(f\left(x_{1}, \cdots, x_{n}\right), y_{1}, \cdots, y_{m}\right)$.) Then $R_{A^{*}(A)}^{\prime}\left(X_{1}, \cdots, X_{n}, Y_{1}, \cdots, Y_{m}\right) \leftrightarrow R_{A^{*}(A)}\left(f_{A^{*}(A)}\left(X_{1}, \cdots, X_{n}\right), Y_{1}, \cdots, Y_{m}\right)$. Combining now we get $\left(X_{1}, \cdots, X_{n}, Y_{1}, \cdots, Y_{m}\right) \notin R_{A^{*}(A)}^{\prime} \cup \bar{R}_{A^{*}(A)}^{\prime}$. Since $X_{1}, \cdots, X_{n}, Y_{1}, \cdots, Y_{m} \in S$, this contradicts the indecomposability of $S$. Hence $S^{\prime}$ is indecomposable and since $S$ is maximal, $S^{\prime}=S$ and $X \in S$. So $S$ is closed under extended arithmetic functions.

The theory of arithmetic has definable Skolem functions. So every statement of arithmetic is equivalent to a universal statement in which the matrix is in disjunctive normal form, $\vee \wedge P$, where $P$ is of the form $u+v=w, u \cdot v=w$, or $u \neq v$ and $u, v, w$ can be variables, integers, or arithmetic Skolem functions of variables. 
Suppose $\varphi$ is a true statement of arithmetic. We claim $\varphi$ is true in $S$, with Skolem functions which are the extensions to $\Lambda^{*}(A)$ of the arithmetic Skolem functions for $\varphi$. $\varphi$ has the form $\left(x_{1}\right) \cdots\left(x_{n}\right)\left[\mathbf{V}_{i=1}^{m} R_{i}\right]$ where each $R_{i}$ is a conjunction of $P$ 's as described above. So assume $X_{1}, \cdots, X_{n} \in S$. Suppose $\left(X_{1}, \cdots, X_{n}\right) \notin\left(R_{i}\right)_{\Lambda^{*}(A)}$ for $i=1$ to $m-1$. Then by the indecomposability of $S,\left(X_{1}, \cdots, X_{n}\right) \in\left(\bar{R}_{i}\right)_{A^{*}(A)}$ for $i=1$ to $m-1$ and thus $\left(X_{1}, \cdots, X_{n}\right) \in\left(\bigcap_{i=1}^{m-1} \bar{R}_{i}\right)_{A^{*}(A)}$. But because $\varphi$ is true in $E^{*}$, we have $\bigcap_{i=1}^{m-1} \bar{R}_{i} \subseteq R_{m}$. Thus $\left(X_{1}, \cdots, X_{n}\right) \in\left(R_{m}\right)_{A^{*}(A)}$. Hence there is some $R_{i}$, call it $R$, such that $\left(X_{1}, \cdots, X_{n}\right) \in R_{A^{*}(A)}$. Let $P$ be one of the conjuncts in $R$. Then $\left(X_{1}, \cdots, X_{n}\right) \in P_{\Lambda^{*}(A)}$. But a triple from $\Lambda^{*}(A)$ which is in the extension of the addition relation in $E^{*}$ satisfies the addition relation in $\Lambda^{*}(A)$; similarly for multiplication and inequality. And since extension commutes with composition, each $P$ in $R$ is satisfied in $\Lambda^{*}(A)$ when $X_{i}$ is substituted for the variable $x_{i}$. But by the earlier part of this proof, $f_{A^{*}(A)}\left(X_{1}, \cdots, X_{n}\right) \in S$ for the Skolem function $f\left(x_{1}, \cdots, x_{n}\right)$. Thus $\varphi$ is true in $S$. Since $\varphi$ was any statement true in arithmetic we have the converse and hence $S$ is an elementary extension of $E^{*}$, as required.

Suppose $S$ is a maximal indecomposable subset of $\Lambda^{*}(A)$. Let $T$ be a subset of $S, T \supseteqq E^{*}$, and let $\bar{T}$ be the closure of $T$ in $S$ under arithmetic functions extended to $\Lambda^{*}(A)$ ( $S$ being closed under such functions). From the proof of the theorem, these functions were exactly the Skolem functions for $S$. Hence $\bar{T}$ is an elementary extension of $E^{*}$ and $\bar{T}$ and $S$ are elementarily equivalent. (Clearly $\bar{T}$ is contained in every maximal indecomposable $S$ which contains $T$.) In particular, if $X \in \Lambda^{*}(A)$ is indecomposable, then

$\left\{f_{d^{*}(A)}(X) \mid f\right.$ is a one-place arithmetic function $\}$

is an elementary extension of $E^{*}$, which is $E^{*}$ if $X$ is finite and which properly extends $E^{*}$ if $X \in \Lambda^{*}(A)-E^{*}$.

The author wishes to acknowledge valuable conversations on the subject of this paper with Professor A. Nerode. He also acknowledges helpful suggestions from the referee.

\section{REFERENCES}

1. S. Feferman and R. L. Vaught, The first order properties of products of algebraic systems, Fund. Math. 47 (1959), 57-103.

2. B. Jónsson and P. Olin, Almost direct products and saturation, Compositio Math. 20 (1968), 125-132.

3. A. Nerode, Extensions to isols, Ann. of Math. 73 (1961), 362-403.

4. —- Extensions to isolic integers, Ann. of Math. 75 (1962), 419-448.

5. - Arithmetically isolated sets and nonstandard models, Amer. Math. Soc. Proc. of Symp. in Pure Math. 5 (1962), 105-116. 
6. - Diophantine correct nonstandard models in the isols, Ann. of Math. 84 (1966), 421-432.

7. P. Olin, An almost everywhere direct power, Trans. Amer. Math. Soc. 134 (1968), 405-420.

Received May 13, 1968. This work was partially supported by the United States Air Force under contract AFOSR-68-1402.

CORNELL UNIVERSITY

ITHACA, NEW YORK 



\section{Pacific Journal of Mathematics \\ Vol. 29, No. 1 \\ May, 1969}

Jorge Alvarez de Araya, A Radon-Nikodým theorem for vector and operator

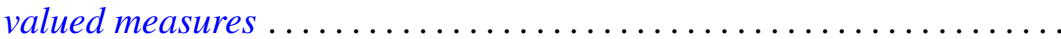

Deane Eugene Arganbright, The power-commutator structure of finite

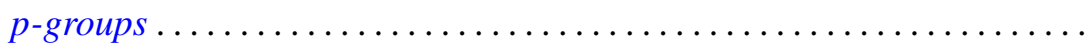

Richard Eugene Barlow, Albert W. Marshall and Frank Proschan, Some

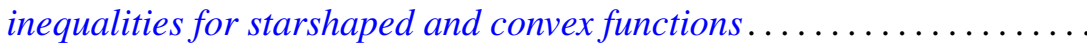

David Clarence Barnes, Some isoperimetric inequalities for the eigenvalues

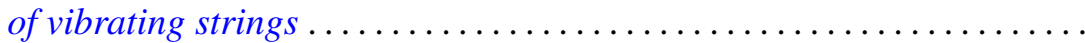

David Hilding Carlson, Critical points on rim-compact spaces ...........

Allan Matlock Weber Carstens, The lattice of pretopologies on an arbitrary

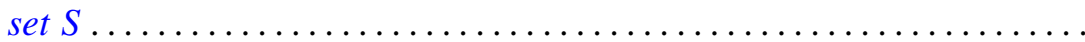

S. K. Chatterjea, A bilateral generating function for the ultraspherical

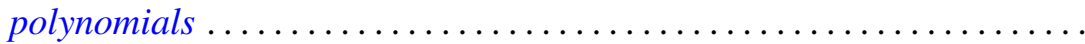

Ronald J. Ensey, Primary Abelian groups modulo finite groups ......... 77

Harley M. Flanders, Relations on minimal hypersurfaces ............ 83

Allen Roy Freedman, On asymptotic density in n-dimensions........... 95

Kent Ralph Fuller, On indecomposable injectives over artinian rings...... 115

George Isaac Glauberman, Normalizers of p-subgroups in finite groups . . . 137

William James Heinzer, On Krull overrings of an affine ring ........... 145

John McCormick Irwin and Takashi Ito, A quasi-decomposable abelian group without proper isomorphic quotient groups and proper isomorphic subgroups.

Allan Morton Krall, Boundary value problems with interior point boundary conditions

John S. Lowndes, Triple series equations involving Laguerre

polynomials

Philip Olin, Indefinability in the arithmetic isolic integers

Ki-Choul Oum, Bounds for the number of deficient values of entire functions whose zeros have angular densities..

R. D. Schafer, Standard algebras ....................

Wolfgang M. Schmidt, Irregularities of distribution. III.

Richard Alfred Tapia, An application of a Newton-like method to the Euler-Lagrange equation 